\title{
Common Fixed Point Theorems for Finite Number of Mappings without Continuity and Compatibility on Fuzzy Metric Spaces
}

\author{
Sushil Sharma and Bhavana Deshpande
}

\begin{abstract}
The aim of this paper is to prove some common fixed point theorems for finite number of discontinuous, noncompatible mappings on noncomplete fuzzy metric spaces. We improve extend and generalize several fixed point theorems on metric spaces, uniform spaces and fuzzy metric spaces. We also give formulas for total number of commutativity conditions for finite number of mappings
\end{abstract}

\section{INTRODUCTION AND PRELIMINARIES}

There have been several attempts to formulate fixed point theorems in fuzzy mathematics after investigation of the notion of fuzzy sets by Zadeh [35]. From amongst several formulations of fuzzy metric spaces, Grabiec [4] followed Kramosil and Michalek [12] and obtained fuzzy version of Banach contraction principle.

The notion of weak commutativity as an improvement of commutativity was introduced by Sessa [22]. Jungck [8] enlarged this concept to compatibility and later weak compatibility [11].

The notion of compatible maps in fuzzy metric spaces has been introduced by Mishra et. al [13], compatible maps of type $(\alpha)$ by Cho [1] and compatible maps of type $(\beta)$ by Cho, Pathak, Kang and Jung [2].

Mishra, Sharma and Singh [13] extended, generalized and fuzzified several known fixed point theorems for contractive type maps on metric and other spaces by using condition of compatibility and continuity of

2000 Mathematics Subject Classification. Primary: 47H10, 54H25.

Key words and phrases. Fuzzy metric spaces, Noncompatible mappings, Common fixed points. 
one map in each compatible pair to prove fixed point theorem for four maps in complete fuzzy metric spaces.

There after many authors proved the result for four, five or six mappings in complete fuzzy metric spaces by using either condition of compatibility or compatibility of type $(\alpha)$ or compatible maps of type $(\beta)$ and continuity of two or more mappings. (Cho [1], Cho, Pathak, Kang and Jung [2], Sharma [24, 25], Sharma and Deshpande [26] and many others).

Number of these theorems are very useful but their hypothesis are very difficult to satisfy as they require continuity and compatibility of involved mappings.

There are so many functions which are not continuous but have a fixed point.

For example the function $f$ defined on $R$ by

$$
f(x)= \begin{cases}0, & x \leq 0 \\ 1, & x>0\end{cases}
$$

This function $f$ is not continuous at 0 but has 0 as a fixed point.

Another example is Dirichlet function defined on $R$ by

$$
f(x)= \begin{cases}1, & \text { if } x \text { is rational, } \\ 0, & \text { if } x \text { is irrational }\end{cases}
$$

Dirichlet function is not continuous at any point but has 1 as a fixed point.

These observations motivated several authors of the field to prove fixed point theorems for noncompatible, discontinuous mappings.

Pant $[14,15,16,17]$ initiated the study of noncompatible maps and introduced the notion of $R$-weakly commuting maps in [14]. He showed that compatible maps are $R$-weakly commuting but converse need not true.

Vasuki [34] defined $R$-weak commutativity in fuzzy metric spaces.

Sharma and Deshpande [27] proved fixed point theorem for four mappings without assuming continuity, any type of compatibility and any commutativity condition in complete fuzzy metric spaces.

In their papers, Sharma and Deshpande [28, 29] extended, improved, generalized and fuzzified several results by proving fixed point theorems for five and six mappings without assuming continuity and any type of compatibility in noncomplete fuzzy metric spaces.

In this paper, we prove a common fixed point theorem for ten noncompatible, discontinuous mappings in noncomplete fuzzy metric spaces. 
We also extend our results for finite number of mappings. We improve, extend and generalize several fixed point theorems on metric spaces, Menger probabilistic metric spaces, uniform spaces and fuzzy metric spaces $([1,2,4,5,6,7,8,9,10,13,24,25,26,27,28,29,30,31,32,33])$.

To prove existence of common fixed point for finite number of mappings some commutativity conditions are required. How many commutativity conditions are required? We give answer of this question by giving some formulas.

Definition 1 ([21]). A binary operation $*:[0,1] \times[0,1] \rightarrow[0,1]$ is continuous $t$-norm if $\{[0,1], *\}$ is an Abelian topological monoid with unit 1 such that $a * b \leq c * d$ whenever $a \leq c$ and $b \leq d, a, b, c, d \in[0,1]$.

Examples of $t$-norm are $a * b=\min \{a, b\}, a * b=a \cdot b$ and $a * b=$ $\max \{a+b-1\}$.

Definition $2([3])$. The 3 -tuple $(X, M, *)$ is called a fuzzy metric space if $X$ is an arbitrary set, $*$ is a continuous $t$-norm and $M$ is a fuzzy set in $X^{2} \times[0, \infty)$ satisfying the following conditions for all $x, y, z \in X$ and $t, s>0$ :

(i) $M(x, y, t)>0$;

(ii) $M(x, y, t)=1$ for all $t>0$ if and only if $x=y$;

(iii) $M(x, y, t)=M(y, x, t)$;

(iv) $M(x, y, t) * M(y, z, s) \leq M(x, z, t+s)$;

(v) $M(x, y, \bullet):[0, \infty) \rightarrow[0,1]$ is continuous.

In this paper $(X, M, *)$ will denote a fuzzy metric space in the sense of the above definition with the following condition:

(vi) $\lim _{t \rightarrow \infty} M(x, y, t)=1$ for all $x, y$ in $X$.

Note that $M(x, y, t)$ can be thought as the degree of nearness between $x$ and $y$ with respect to $t$. We identify $x=y$ with $M(x, y, t)=1$ for all $t>0$ and $M(x, y, t)=0$ with $\infty$ and we can find some topological properties and examples of fuzzy metric spaces in [3].

In the following example, we know that every metric induces a fuzzy metric.

Example $1([3])$. Let $(X, d)$ be a metric space. Define $a * b=a b$ or $a * b=\min \{a, b\}$ and for all $x, y \in X, t>0$,

$$
M(x, y, t)=\frac{t}{t+d(x, y)} .
$$

Then $(X, M, *)$ is a fuzzy metric space. We call this fuzzy metric $M$ induced by the metric $d$ the standard fuzzy metric. 
Lemma 1 ([4]). For all $x, y \in X, M(x, y, \cdot)$ is nondecreasing.

Definition $3([4])$. A sequence $\left\{x_{n}\right\}$ in a fuzzy metric space $(X, M, *)$ is called Cauchy if $\lim _{n \rightarrow \infty} M\left(x_{n+p}, x_{n}, t\right)=1$ for every $t>0$ and each $p>0 .(X, M, *)$ is complete if every Cauchy sequence in $X$ converges in $X$. A sequence $\left\{x_{n}\right\}$ in $X$ converges to $x \in X$ if $\lim _{n \rightarrow \infty} M\left(x_{n}, x, t\right)=1$ for every $t>0$.

Lemma $2([1])$. Let $\left\{y_{n}\right\}$ be a sequence in fuzzy metric space $(X, M, *)$. If there exists a number $k \in(0,1)$ such that

$$
M\left(y_{2 n+2}, y_{2 n+1}, k t\right) \geq M\left(y_{2 n+1}, y_{2 n}, t\right)
$$

for all $t>0$ and $n=1,2, \ldots$ then $\left\{y_{n}\right\}$ is a Cauchy sequence in $X$.

Lemma 3 ([13]). If for all $x, y \in X, t>0$ and for a number $k \in(0,1)$

$$
M(x, y, k t) \geq M(x, y, t),
$$

then $x=y$.

Definition $4([15])$. Let $(X, M, *)$ be a fuzzy metric space and let $A, B$ be self mappings of $X$. The mappings $A$ and $B$ are said to be compatible if

$$
\lim _{n \rightarrow \infty} M\left(A B x_{n}, B A x_{n}, t\right)=1,
$$

for all $t>0$, whenever $\left\{x_{n}\right\}$ is a sequence in $X$ such that

$$
\lim _{n \rightarrow \infty} A x_{n}=\lim _{n \rightarrow \infty} B x_{n}=z
$$

for some $z \in X$.

Definition 5 ([11]). Two self maps $A$ and $B$ on a set $X$ are said to be weakly compatible if they commute at coincidence point.

Remark 1. (i) In $[8,10,18]$ we can find the equivalent formulations of definitions of compatible maps, compatible maps of type $(\alpha)$ and compatible maps of type $(\beta)$. Such maps are independent of each other and more general than commuting and weakly commuting maps $([7,22])$.

(ii) Compatible or compatible of type $(\alpha)$ or compatible of type $(\beta)$ maps are weakly compatible but converse need not true [28].

On the lines of Pant [14] now we define pointwise $R$-weak commutativity of mappings in fuzzy metric spaces:

Definition 6. Two self mappings $A$ and $B$ of a fuzzy metric space are called $R$-weakly commuting at a point $x$ in $X$ if

$$
M(A B x, B A x, t) \geq M\left(A x, B x, \frac{t}{R}\right)
$$


for some $R>0$.

The mappings $A$ and $B$ are called pointwise $R$-weakly commuting if given $x$ in $X$ there exists $R>0$ such that

$$
M(A B x, B A x, t) \geq M\left(A x, B x, \frac{t}{R}\right) .
$$

Definition 7 ([34]). Let $(X, M, *)$ be a fuzzy metric space and let $A, B$ be self mappings of $X$. The mappings $A$ and $B$ are said to be $R$-weakly commuting if there exists a positive real number $R$ such that

$$
M(A B x, B A x, t) \geq M\left(A x, B x, \frac{t}{R}\right)
$$

for all $x$ in $X$.

Remark $2([14,15])$. (i) Pointwise $R$-weak commutativity is a necessary, hence minimal condition for the existence of common fixed points of contractive type maps.

(ii) Compatible mappings are necessarily pointwise $R$-weakly commuting. However pointwise $R$-weakly commuting maps need not be compatible.

(iii) Weak compatibility of $A$ and $B$ is equivalent to $R$-weak commutativity of $A$ and $B$ at their coincidence points.

In our theorems and corollaries $(X, M, *)$ will denote fuzzy metric space ( $F M$-space) with

$$
t * t \geq t \quad \text { for all } t \in[0,1] .
$$

2. Main Results

Theorem 1. Let $(X, M, *)$ be an FM-space. Let $A, B, S, T, I, J$, $L, U, P$ and $Q$ be mappings from $X$ into itself such that

(1.1) $P(X) \subset A B I L(X), \quad Q(X) \subset S T J U(X)$;

(1.2) there exists a constant $k \in(0,1)$ such that

$$
\begin{aligned}
{[1+} & a M(S T J U x, A B I L y, k t)] * M(P x, Q y, k t) \\
\geq & a[M(P x, S T J U x, k t) * M(Q y, A B I L y, k t) \\
& +M(Q y, S T J U x, k t) * M(P x, A B I L y, k t)] \\
& +M(A B I L y, S T J U x, t) * M(P x, S T J U x, t) * M(Q y, A B I L y, t) \\
& * M(Q y, S T J U x, \alpha t) * M(P x, A B I L y,(2-\alpha) t) \\
& \quad \text { for all } x, y \in X, a \geq 0, \alpha \in(0,2) \text { and } t>0 ; \\
(1.3) \text { if one of } P(X), A B I L(X), S T J U(X), Q(X) \text { is a complete } & \text { subspace of } X \text { then }
\end{aligned}
$$

(i) $P$ and STJU have a coincidence point; and 
(ii) $Q$ and $A B I L$ have a coincidence point.

Further if

(1.4) $A B=B A, A I=I A, A L=L A, B I=I B, B L=L B, I L=$ $L I, Q L=L Q, Q I=I Q, Q B=B Q, S T=T S, S J=J S$, $S U=U S, T J=J T, T U=U T, J U=U J, P U=U P$, $P J=J P, P T=T P$;

(1.5) the pairs $\{P, S T J U\}$ and $\{Q, A B I L\}$ are weakly compatible, then

(iii) $A, B, S, T, I, J, L, U, P$ and $Q$ have a unique common fixed point in $X$.

Proof. By (1.1) since $P(X) \subset A B I L(X)$ for any point $x_{0} \in X$ there exists a point $x_{1}$ in $X$ such that $P x_{0}=A B I L x_{1}$. Since $Q(X) \subset$ $\operatorname{STJU}(X)$, for this point $x_{1}$ we can choose a point $x_{2}$ in $X$ such that $Q x_{1}=S T J U x_{2}$ and so on. Inductively, we can define a sequence $\left\{y_{n}\right\}$ in $X$ such that for $n=0,1,2, \ldots$

$y_{2 n}=P x_{2 n}=A B I L x_{2 n+1} \quad$ and $\quad y_{2 n+1}=Q x_{2 n+1}=S T J U x_{2 n+2}$.

By (1.2), for all $t>0$ and $\alpha=1-q$ with $q \in(0,1)$, we have

$\left[1+a M\left(y_{2 n}, y_{2 n+1}, k t\right)\right] * M\left(y_{2 n+1}, y_{2 n+2}, k t\right)$

$\geq a\left[M\left(y_{2 n+2}, y_{2 n+1}, k t\right) * M\left(y_{2 n+1}, y_{2 n}, k t\right)\right.$

$\left.+M\left(y_{2 n+1}, y_{2 n+1}, k t\right) * M\left(y_{2 n+2}, y_{2 n}, k t\right)\right]$

$+M\left(y_{2 n}, y_{2 n+1}, t\right) * M\left(y_{2 n+2}, y_{2 n+1}, t\right) * M\left(y_{2 n+1}, y_{2 n}, t\right)$

$* M\left(y_{2 n+1}, y_{2 n+1},(1-q) t\right) * M\left(y_{2 n+2}, y_{2 n},(1+q) t\right)$

$\geq a\left[M\left(y_{2 n}, y_{2 n+1}, k t\right) * M\left(y_{2 n+1}, y_{2 n+2}, k t\right)\right]$

$+M\left(y_{2 n}, y_{2 n+1}, t\right) * M\left(y_{2 n+1}, y_{2 n+2}, t\right) * M\left(y_{2 n}, y_{2 n+1}, q t\right)$.

Thus it follows that

$$
\begin{aligned}
& M\left(y_{2 n+1}, y_{2 n+2}, k t\right) \geq M\left(y_{2 n}, y_{2 n+1}, t\right) \\
& \quad * M\left(y_{2 n+1}, y_{2 n+2}, t\right) * M\left(y_{2 n}, y_{2 n+1}, q t\right) .
\end{aligned}
$$

Since the $t$-norm $*$ is continuous and $M(x, y, \cdot)$ is continuous, letting $q \rightarrow 1$, we have

$$
M\left(y_{2 n+1}, y_{2 n+2}, k t\right) \geq M\left(y_{2 n}, y_{2 n+1}, t\right) * M\left(y_{2 n+1}, y_{2 n+2}, t\right) .
$$

Similarly, we also have

$$
M\left(y_{2 n+2}, y_{2 n+3}, k t\right) \geq M\left(y_{2 n+1}, y_{2 n+2}, t\right) * M\left(y_{2 n+2}, y_{2 n+3}, t\right) .
$$

In general, we have for $m=1,2, \ldots$

$$
M\left(y_{m+1}, y_{m+2}, k t\right) \geq M\left(y_{m}, y_{m+1}, t\right) * M\left(y_{m+1}, y_{m+2}, t\right) .
$$


Consequently, it follows that for $m=1,2, \ldots, p=1,2, \ldots$

$$
M\left(y_{m+1}, y_{m+2}, k t\right) \geq M\left(y_{m}, y_{m+1}, t\right) * M\left(y_{m+1}, y_{m+2}, t / k^{p}\right) .
$$

By noting that $M\left(y_{m+1}, y_{m+2}, t / k^{p}\right) \rightarrow 1$ as $p \rightarrow \infty$, we have for $m=1,2, \ldots$

$$
M\left(y_{m+1}, y_{m+2}, k t\right) \geq M\left(y_{m}, y_{m+1}, t\right) .
$$

Hence by Lemma 2, $\left\{y_{n}\right\}$ is a Cauchy sequence in $X$. Now suppose $\operatorname{STJU}(X)$ is complete. Note that the subsequence $\left\{y_{2 n+1}\right\}$ is contained in $S T J U(X)$ and has a limit in $S T J U(X)$ call it $z$. Let $w \in S T J U^{-1}(z)$. Then $S T J U w=z$. We shall use the fact that subsequence $\left\{y_{2 n}\right\}$ also converges to $z$. By (1.2), with $\alpha=1$ we have

By putting $x=w, y=x_{2 n+1}$ in (1.2) with $\alpha=1$ and taking limit as $n \rightarrow \infty$ we have

$$
M(P w, z, k t) \geq M(P w, z, t) .
$$

Therefore by Lemma 3, we have $P w=z$. Since $S T J U w=z$ thus we have $P w=z=S T J U w$ that is $w$ is coincidence point of $P$ and STJU. This proves (i).

Since $\mathrm{P}(\mathrm{X}) \subset \mathrm{ABIL}(\mathrm{X}), \mathrm{Pw}_{\mathrm{w}}=\mathrm{z}$ implies that $\mathrm{z} \in \mathrm{ABIL}(\mathrm{X})$. Let $v \in A B I L^{-1} z$. Then $A B I L v=z$.

By putting $x=x_{2 n+2}, y=v$ in (1.2), with $\alpha=1$ and taking limit as $n \rightarrow \infty$ we have

$$
M(Q v, z, k t) \geq M(Q v, z, t) .
$$

Therefore by Lemma 3, we have $Q v=z$. Since $A B I L v=z$, we have $Q v=z=A B I L v$ that is $v$ is coincidence point of $Q$ and $A B I L$. This proves (ii).

The remaining two cases pertain essentially to the previous cases. Indeed if $P(X)$ or $Q(X)$ is complete then by (1.1), $z \in P(X) \subset$ $A B I L(X)$ or $z \in Q(X) \subset S T J U(X)$. Thus (i) and (ii) are completely established.

Since the pair $\{P, S T J U\}$ is weakly compatible therefore $P$ and STJU commute at their coincidence point that is $P(S T J U w)=$ $(S T J U) P w$ or $P z=S T J U z$.

Since the pair $\{Q, A B I L\}$ is weakly compatible therefore $Q$ and $A B I L$ commute at their coincidence point that is $Q(A B I L v)=$ $(A B I L) Q v$ or $Q z=A B I L z$.

By putting $x=z, y=x_{2 n+1}$ in (1.2) with $\alpha=1$ and taking limit as $n \rightarrow \infty$ we have

$$
M(P z, z, k t) \geq M(P z, z, t) .
$$


Therefore by Lemma 3 , we have $P z=z$. So $P z=S T J U z=z$. By putting $x=x_{2 n+2}, y=z$ in (1.2) with $\alpha=1$ and taking limit as $n \rightarrow \infty$ we have

$$
M(z, Q z, k t) \geq M(Q z, z, t) .
$$

Therefore by Lemma 3, we have $Q z=z$, so $Q z=A B I L z=z$. By putting $x=z, y=L z$ in (1.2), with $\alpha=1$ and using (1.4), we have $M(z, L z, k t) \geq M(L z, z, t) * 1 * 1 * M(L z, z, t) * M(L z, z, t) \geq M(L z, z, t)$

Therefore by Lemma 3 , we have $L z=z$. Since $A B I L z=z$ therefore $A B I z=z$. By putting $x=z, y=I z$ in (1.2), with $\alpha=1$ and using (1.4), we have

$M(I z, z, k t) \geq M(I z, z, t) * 1 * 1 * M(I z, z, t) * M(z, I z, t) \geq M(I z, z, t)$.

Therefore by Lemma 3 , we have $I z=z$. Since $A B I z=z$ therefore $A B z=z$. Now to prove $B z=z$ we put $x=z, y=B z$ in (1.2), with $\alpha=1$ and using (1.4), we have

$$
\begin{aligned}
M(z, B z, k t) & \geq M(B z, z, t) * 1 * 1 * M(B z, z, t) * M(z, B z, t) \\
& \geq M(B z, z, t) .
\end{aligned}
$$

Therefore by Lemma 3 , we have $B z=z$. Since $A B z=z$ therefore $A z=z$. To prove $U z=z$, we put $x=U z, y=z$ in (1.2), with $\alpha=1$ and using (1.4), we have

$$
\begin{aligned}
M(U z, z, k t) & \geq M(U z, z, t) * 1 * 1 * M(U z, z, t) * M(U z, z, t) \\
& \geq M(U z, z, t) .
\end{aligned}
$$

Therefore by Lemma 3 , we have $U z=z$. Since $S T J U z=z$ therefore $S T J z=z$. To prove $J z=z$ put $x=J z, y=z$ in (1.2) with $\alpha=1$ and using (1.4), we have

$$
\begin{aligned}
M(J z, z, k t) & \geq M(J z, z, t) * 1 * 1 * M(J z, z, t) * M(J z, z, t) \\
& \geq M(J z, z, t) .
\end{aligned}
$$

Therefore by Lemma 3, we have $J z=z$. Since $S T J z=z$ therefore $S T z=z$. To prove $T z=z$ put $x=T z, y=z$ in (1.2), with $\alpha=1$ and using (1.4), we have

$$
\begin{aligned}
M(T z, z, k t) & \geq M(T z, z, t) * 1 * 1 * M(T z, z, t) * M(T z, z, t) \\
& \geq M(T z, z, t) .
\end{aligned}
$$

Therefore by Lemma 3, we have $T z=z$. Since $S T z=z$ therefore $S z=z$. By combining the above results we have

$$
A z=B z=S z=T z=I z=J z=L z=U z=P z=Q z=z,
$$


that is $z$ is a common fixed point of $A, B, S, T, I, J, L, U, P$ and $Q$. The uniqueness of the common fixed point of $A, B, S, T, I, J, L, U, P$ and $Q$ follows easily from (1.2). This completes the proof.

From Theorem 1, with $a=0$, we have the following result:

Corollary 2. Let $(X, M, *)$ be an FM-space. Let $A, B, S, T, I, J$, $L, U, P$ and $Q$ be mappings from $X$ into itself satisfy condition (1.2) with $a=0$. If conditions (1.1) and (1.3) are satisfied then conclusions (i) and (ii) of Theorem 1 hold. Further if conditions (1.4) and (1.5) are satisfied then conclusion (iii) of Theorem 1 holds.

If we put $P=Q$ in Theorem 1, we have the following result:

Corollary 3. Let $(X, M, *)$ be an FM-space. Let $A, B, S, T, I, J, L, U$ and $P$ be mappings from $X$ into itself such that

(3.1) $P(X) \subset A B I L(X), P(X) \subset S T J U(X)$;

(3.2) there exists a constant $k \in(0,1)$ such that

$$
\begin{aligned}
{[1+} & a M(S T J U x, A B I L y, k t)] * M(P x, P y, k t) \\
\geq a & {[M(P x, S T J U x, k t) * M(P y, A B I L y, k t)} \\
& +M(P y, S T J U x, k t) * M(P x, A B I L y, k t)] \\
& +M(A B I L y, S T J U x, t) * M(P x, S T J U x, t) \\
& * M(P y, A B I L y, t) * M(P y, S T J U x, \alpha t) \\
& * M(P x, A B I L y,(2-\alpha) t)
\end{aligned}
$$

for all $x, y \in X, a \geq 0, \alpha \in(0,2)$ and $t>0$,

(3.3) if one of $P(X), A B I L(X), S T J U(X)$ is a complete subspace of $X$ then

(i) $P$ and STJU have a coincidence point; and

(ii) $P$ and $A B I L$ have a coincidence point.

Further if

(3.4) $A B=B A, A I=I A, A L=L A, B I=I B, B L=L B, I L=$ $L I, P L=L P, P I=I P, P B=B P, S T=T S, S J=J S$, $S U=U S, T J=J T, T U=U T, J U=U J, P U=U P$, $P J=J P, P T=T P$

(3.5) the pairs $\{P, S T J U\}$ and $\{P, A B I L\}$ are weakly compatible, then

(iii) $A, B, S, T, I, J, L, U$ and $P$ have a unique common fixed point in $X$.

From Corollary 3 with $a=0$, we have the following: 
Corollary 4. Let $(X, M, *)$ be an FM-space. Let $A, B, S, T, I, J, L, U$ and $P$ be mappings from $X$ into itself satisfy condition (3.2) with $a=0$. If conditions (3.1) and (3.3) are satisfied then conclusions (i) and (ii) of Corollary 3 hold. Further if conditions (3.4) and (3.5) are satisfied then conclusion (iii) of Corollary 3 holds.

If we put $L=U=I_{X}$ (the identity map on $X$ ) in Theorem 1, we have the following:

Corollary 5. Let $(X, M, *)$ be an FM-space. Let $A, B, S, T, I, J, P$ and $Q$ be mappings from $X$ into itself such that

(5.1) $P(X) \subset A B I(X), Q(X) \subset S T J(X)$;

(5.2) there exists a constant $k \in(0,1)$ such that

$$
\begin{aligned}
{[1+} & a M(S T J x, A B I y, k t)] * M(P x, Q y, k t) \\
\geq a & {[M(P x, S T J x, k t) * M(Q y, A B I y, k t)} \\
+ & M(Q y, S T J x, k t) * M(P x, A B I y, k t)] \\
+ & M(A B I y, S T J x, t) * M(P x, S T J x, t) \\
& * M(Q y, A B I y, t) * M(Q y, S T J x, \alpha t) \\
& * M(P x, A B I y,(2-\alpha) t)
\end{aligned}
$$

for all $x, y \in X, a \geq 0, \alpha \in(0,2)$ and $t>0$;

(5.3) if one of $P(X), A B I(X), S T J(X), Q(X)$ is a complete subspace of $X$ then

(i) $P$ and $S T J$ have a coincidence point; and

(ii) $Q$ and $A B I$ have a coincidence point.

Further if

(5.4) $A B=B A, A I=I A, B I=I B, Q I=I Q, Q B=B Q$, $S T=T S, S J=J S, T J=J T, P J=J P, P T=T P ;$

(5.5) the pairs $\{P, S T J\}$ and $\{Q, A B I\}$ are weakIy compatibIe, then (iii) $A, B, S, T, I, J, P$ and $Q$ have a unique common fixed point in $X$.

If we put $a=0$ in Corollary 5 , we get the following:

Corollary 6. Let $(X, M, *)$ be an FM-space. Let $A, B, S, T, I, J, P$ and $Q$ be mappings from $X$ into itself satisfy condition (5.2) with $a=0$. If conditions (5.1) and (5.3) are satisfied then conclusions (i) and (ii) of Corollary 5 hold. Further if conditions (5.4) and (5.5) are satisfied then conclusion (iii) of Corollary 5 holds.

If we put $P=Q$ in Corollary 5 we get the following: 
Corollary 7. Let $(X, M, *)$ be an FM-space. Let $A, B, S, T, I, J$ and $P$ be mappings from $X$ into itself such that

(7.1) $P(X) \subset A B I(X), P(X) \subset S T J(X)$;

(7.2) there exists a constant $k \in(0,1)$ such that

$$
\begin{aligned}
& {[1+a M(S T J x, A B I y, k t)] * M(P x, P y, k t)} \\
& \geq a[M(P x, S T J x, k t) * M(P y, A B I y, k t)+M(P y, S T J x, k t) \\
& \quad * M(P x, A B I y, k t)]+M(A B I y, S T J x, t) * M(P x, S T J x, t) \\
& \quad * M(P y, A B I y, t) * M(P y, S T J x, \alpha t) * M(P x, A B I y,(2-\alpha) t) \\
& \quad \text { for all } x, y \in X, a \geq 0, \alpha \in(0,2) \text { and } t>0,
\end{aligned}
$$

(7.3) if one of $P(X), A B I(X), S T J(X)$ is a complete subspace of $X$ then

(i) $P$ and STJ have a coincidence point; and

(ii) $P$ and $A B I$ have a coincidence point.

Further if

(7.4) $A B=B A, A I=I A, B I=I B, P I=I P, P B=B P$, $S T=T S, S J=J S, T J=J T, P J=J P, P T=T P$;

(7.5) the pairs $\{P, S T J\}$ and $\{P, A B I\}$ are weakly compatible, then (iii) $A, B, S, T, I, J$ and $P$ have a unique common fixed point in $X$.

If we put $a=0$ in Corollary 7 , we get the following:

Corollary 8. Let $(X, M, *)$ be an FM-space. Let $A, B, S, T, I, J$ and $P$ be mappings from $X$ into itself satisfy condition (7.2) with $a=0$. If conditions (7.1) and (7.3) are satisfied then conclusions (i) and (ii) of Corollary 7 hold. Further if condition (7.4) and (7.5) are satisfied then conclusion (iii) of Corollary 7 holds.

Remark 3. Theorem 1 and Corollary 2-8 improve, extend and generalize the results of Cho [1], Cho, Pathak, Kang and Jung [2], Iseki [5], Istratescu [6], Jungck [7, 8, 9], Jungck, Murthy and Cho [10], Mishra, Sharma and Singh [13], Rhoades [19, 20], Sharma [24, 25], Sharma and Deshpande [26, 27, 28, 29], Singh [30], Singh and Kasahara [31], Sing and Ram [32], Tiwari and Singh [33].

Remark 4. (i) From Corollary 5, with $I=J=I_{X}$ (the identity map on $X$ ) we obtain the result due to Sharma and Deshpande [29].

(ii) From Corollary 5, with $a=0$ and $I=J=I_{X}$ (the identity map on $X$ ) we obtain the result due to Sharma and Deshpande $[28]$. 
(iii) From Corollary 5, with $P=Q$ and $I=J=I_{X}$ (the identity map on $X$ ) we obtain the result due to Sharma and Deshpande [29].

(iv) From Corollary 5, with $a=0, P=Q$ and $I=J=I_{X}$ (the identity map on $X$ ) we obtain the result due to Sharma and Deshpande [28].

(v) From Corollary 5 with $B=T=I=J=I_{X}$ (the identity map on $X$ ) we obtain the result due to Sharma and Deshpande [29].

(vi) From Corollary 5 with $a=0, B=T=I=J=I_{X}$ (the identity map on $X$ ) we obtain the result due to Sharma and Deshpande [28].

Example 2. Let $X=[0,15)$ with the metric $d$ defined by $d(x, y)=$ $|x-y|$. For each $t \in(0, \infty)$ define

$$
\begin{array}{ll}
M(x, y, t)=\frac{t}{t+d(x, y)}, & x, y \in X, \\
M(x, y, 0)=0, & x, y \in X .
\end{array}
$$

Then $(X, M, *)$ is a fuzzy metric space where $*$ is defined by $a * b=$ $a \cdot b$. Clearly $(X, M, *)$ is a noncomplete fuzzy metric space. Define $A, S, P$ and $Q: X \rightarrow X$ by

$$
\begin{aligned}
& P x=\left\{\begin{array}{lll}
0, & \text { if } & x=0 \\
1.5, & \text { if } & x>0
\end{array}\right. \\
& Q x=\left\{\begin{array}{lll}
0, & \text { if } & x=0 \\
3.5, & \text { if } & x>0 .
\end{array}\right. \\
& A x=\left\{\begin{array}{lll}
0, & \text { if } x=0 \\
1.5, & \text { if } 0<x \leq 5 \\
x-1.5, & \text { if } x>5
\end{array}\right. \\
& S x=\left\{\begin{array}{lll}
0, & \text { if } x=0 \\
3, & \text { if } 0<x \leq 5 \\
x-3.5, & \text { if } x>5 .
\end{array}\right.
\end{aligned}
$$

If we take $t=1, k=0.5$ and $\alpha=1$ we see that $A, S, P$ and $Q$ satisfy all the conditions of Remark 4(v) and Remark 4(vi) and have a unique common fixed point $0 \in X$. It may be noted in this example that the mappings $P$ and $S$ commute at coincidence point $0 \in X$. So $P$ and $S$ are weakly compatible maps. Similarly $Q$ and $A$ are weakly compatible maps. To see the pairs $\{P, S\}$ and $\{Q, A\}$ are 
noncompatible. Let us consider a decreasing sequence $\left\{x_{n}\right\}$ such that $x_{n} \rightarrow 5$. Then $\lim _{n \rightarrow \infty} P x_{n}=1.5, \lim _{n \rightarrow \infty} S x_{n}=1.5$ but

$$
\lim _{n \rightarrow \infty} M\left(P S x_{n}, S P x_{n}, t\right)=\frac{t}{t+|1.5-3.0|} \neq 1 .
$$

Thus the pair $\{P, S\}$ is noncompatible. Also

$$
\lim _{n \rightarrow \infty} Q x_{n}=3.5, \quad \lim _{n \rightarrow \infty} A x_{n}=3.5
$$

but

$$
\lim _{n \rightarrow \infty} M\left(Q A x_{n}, A Q x_{n}, t\right)=\frac{t}{t+|3.5-1.5|} \neq 1 .
$$

So the pair $\{Q, A\}$ is noncompatible. It can be easily verified in this example that the pairs $\{P, S\}$ and $\{Q, A\}$ are neither compatible of type $(\alpha)$ nor compatible of type $(\beta)$. All the mappings involved in this example are discontinuous even at the common fixed point $x=0$.

If we put $A=S, B=T=I=J=I_{X}$ (the identity map on $X$ ) in Corollary 5 , we have the following result:

Corollary 9. Let $(X, M, *)$ be an FM-space. Let $A, P$ and $Q$ be mappings from $X$ into itself such that

(9.1) $P(X) \subset A(X), Q(X) \subset A(X)$;

(9.2) there exists a constant $k \in(0,1)$ such that

$$
\begin{aligned}
& {[1+a M(A x, A y, k t)] * M(P x, Q y, k t)} \\
& \geq a[M(P x, A x, k t) * M(Q y, A y, k t)+M(Q y, A x, k t) \\
& \quad * M(P x, A y, k t)]+M(A y, A x, t) * M(P x, A x, t) \\
& \quad * M(Q y, A y, t) * M(Q y, A x, \alpha t) * M(P x, A y,(2-\alpha) t)
\end{aligned}
$$

for all $x, y \in X, a \geq 0, \alpha \in(0,2)$ and $t>0$;

(9.3) if one of $P(X), A(X), Q(X)$ is a complete subspace of $X$ then

(i) $P$ and $A$ have a coincidence point; and

(ii) $Q$ and $A$ have a coincidence point.

Further if

(9.4) the pairs $\{P, A\}$ and $\{Q, A\}$ are weakIy compatibIe, then

(iii) $A, P$ and $Q$ have a unique common fixed point in $X$.

From Corollary 9, with $a=0$ we have the following:

Corollary 10. Let $(X, M, *)$ be an FM-space. Let $A, P$ and $Q$ be mappings from $X$ into itself satisfy condition (9.2) with $a=0$.

If conditions (9.1) and (9.3) are satisfied then conclusions (i) and (ii) of Corollary 9 hold. Further if conditions (9.4) and (9.5) are satisfied then conclusion (iii) of Corollary 9 holds. 
Remark 5. (i) If we put $A=I_{X}$ (the identity map on $X$ ) in Corollary 9, we obtain the result due to Cho, Pathak, Kang and Jung [2].

If we put $A=I_{X}$ (the identity map on $X$ ) and $a=0$ in Corollary 9 , we have the following result:

Corollary 11. Let $(X, M, *)$ be an $F M$-space. Let $P$ and $Q$ be mappings from $X$ into itself such that

(11.1) there exists a constant $k \in(0,1)$ such that

$$
\begin{aligned}
M(P x, Q y, k t) \geq M(y, x, t) & * M(P x, x, t) * M(Q y, y, t) \\
& * M(Q y, x, \alpha t) * M(P x, y,(2-\alpha) t)
\end{aligned}
$$

for all $x, y \in X, \alpha \in(0,2)$ and $t>0$;

(11.2) if one of $P(X), Q(X)$ is a complete subspace of $X$ then $P$ and $Q$ have a unique common fixed point in $X$.

If we put $P=Q$ in Corollary 11 we have the following:

Corollary 12. Let $(X, M, *)$ be an FM-space. Let $P$ be mapping from $X$ into itself such that

(12.1) there exists a constant $k \in(0,1)$ such that

$$
\begin{aligned}
& {[1+a M(x, y, k t)] * M(P x, P y, k t)} \\
& \geq a[M(P x, x, k t) * M(P y, y, k t)+M(P y, x, k t) \\
& \quad * M(P x, y, k t)]+M(y, x, t) * M(P x, x, t) * M(P y, y, t) \\
& \quad * M(P y, x, \alpha t) * M(P x, y,(2-\alpha) t)
\end{aligned}
$$

for all $x, y \in X, a \geq 0, \alpha \in(0,2)$ and $t>0$;

(12.2) if $P(X)$ is a complete subspace of $X$.

Then $P$ has a unique common fixed point in $X$.

Corollary 13 (Fuzzy Banach contraction theorem [4]). Let $(X, M, *)$ be an FM-space. Let $P$ be mapping from $X$ into itself such that

(13.1) there exists a constant $k \in(0,1)$ such that

$$
M(P x, P y, k t) \geq M(x, y, t)
$$

for all $x, y \in X$ and $t>0$;

(13.2) if $P(X)$ is a complete subspace of $X$.

Then $P$ has a unique common fixed point in $X$.

Proof. It follows from Corollary 12 since (12.1) with $a=0$ includes (13.1). 
In fact, even though we put $s * t=\min \{s, t\}$ in Corollary 12, Corollary 12 is still true and so if we put

$$
\begin{aligned}
M(x, y, t)=\min \{ & M(x, y, t), M(x, P x, t), \\
& M(y, P y, t), M(y, P x, t), M(x, P y, t)\}
\end{aligned}
$$

from (12.1) with $a=0$ we have (13.1).

Remark 6. In Corollary 13, we use condition (13.2) that is $P(X)$ is a complete subspace of $X$, while Grabiec [4] used completeness of the whole space $(X, M, *)$ However Grabiec [4] does not require $t * t \geq t$ in his proof.

Now we extend Theorem 1 for finite number of mappings in the following way:

Theorem 14. Let $(X, M, *)$ be an FM-space. Let $A_{1}, A_{2}, \ldots, A_{n}$, $S_{1}, S_{2}, \ldots, S_{n}, P$ and $Q$ be mappings from $X$ into itself such that

(14.1) $P(X) \subset A_{1} A_{2} \cdots A_{n}(X), Q(X) \subset S_{1} S_{2} \cdots S_{n}(X)$;

(14.2) there exists a constant $k \in(0,1)$ such that

$$
\begin{aligned}
{[1+} & \left.a M\left(S_{1} S_{2} \cdots S_{n} x, A_{1} A_{2} \cdots A_{n} y, k t\right)\right] * M(P x, Q y, k t) \\
\geq & a\left[M\left(P x, S_{1} S_{2} \cdots S_{n} x, k t\right) * M\left(Q y, A_{1} A_{2} \cdots A_{n} y, k t\right)\right. \\
& \left.+M\left(Q y, S_{1} S_{2} \cdots S_{n} x, k t\right) * M\left(P x, A_{1} A_{2} \cdots A_{n} y, k t\right)\right] \\
& +M\left(A_{1} A_{2} \cdots A_{n} y, S_{1} S_{2} \cdots S_{n} x, t\right) * M\left(P x, S_{1} S_{2} \cdots S_{n} x, t\right) \\
& * M\left(Q y, A_{1} A_{2} \cdots A_{n} y, t\right) * M\left(Q y, S_{1} S_{2} \cdots S_{n} x, \alpha t\right) \\
& * M\left(P x, A_{1} A_{2} \cdots A_{n} y,(2-\alpha) t\right)
\end{aligned}
$$

for all $x, y \in X, a \geq 0, \alpha \in(0,2)$ and $t>0$;

(14.3) if one of $P(X), A_{1} A_{2} \cdots A_{n}(X), S_{1} S_{2} \cdots S_{n}(X), Q(X)$ is a complete subspace of $X$ then

(i) $P$ and $S_{1} S_{2} \cdots S_{n}$ have a coincidence point; and

(ii) $Q$ and $A_{1} A_{2} \cdots A_{n}$ have a coincidence point.

Further if

(14.4) $A_{1}$ commutes with $A_{2}, A_{3}, \ldots, A_{n}$,

$A_{2}$ commutes with $A_{3}, A_{4}, \ldots, A_{n}$,

$A_{3}$ commutes with $A_{4}, A_{5}, \ldots, A_{n}$,

$A_{n-1}$ commutes with $A_{n}$, 
$S_{1} \quad$ commutes with $S_{2}, S_{3}, \ldots, S_{n}$,

$S_{2} \quad$ commutes with $S_{3}, S_{4}, \ldots, S_{n}$,

$S_{3} \quad$ commutes with $S_{4}, S_{5}, \ldots, S_{n}$,

$S_{n-1}$ commutes with $S_{n}$,

$P \quad$ commutes with $S_{2}, S_{3}, \ldots, S_{n}$,

$Q \quad$ commutes with $A_{2}, A_{3}, \ldots, A_{n}$;

(14.5) the pairs $\left\{P, S_{1} S_{2} \cdots S_{n}\right\}$ and $\left\{Q, A_{1} A_{2} \cdots A_{n}\right\}$ are weakly compatible, then

(iii) $A_{1}, A_{2}, \ldots, A_{n}, S_{1}, S_{2}, \ldots, S_{n}, P$ and $Q$ have a unique common fixed point in $X$.

Proof. Since $P(X) \subset A_{1} A_{2} \cdots A_{n}(X)$, for any point $x_{0} \in X$ there exists a point $x_{1} \in X$ such that $P x_{0}=A_{1} A_{2} \cdots A_{n} x_{1}$. Since $Q(X) \subset$ $S_{1} S_{2} \cdots S_{n}(X)$, for this point $x_{1}$ we can choose a point $x_{2} \in X$ such that $Q x_{1}=S_{1} S_{2} \cdots S_{n} x_{2}$ and so on.

Inductively, we can define a sequence $\left\{y_{n}\right\}$ in $X$ such that for $n=$ $0,1,2, \ldots$

$$
\begin{aligned}
y_{2 n} & =P x_{2 n}=A_{1} A_{2} \cdots A_{n} x_{2 n+1}, \\
y_{2 n+1} & =Q x_{2 n+1}=S_{1} S_{2} \cdots S_{n} x_{2 n+2} .
\end{aligned}
$$

By using the method of proof of Theorem 1, we can see that conclusions (i), (ii) and (iii) hold.

From Theorem 14, with $a=0$, we have the following:

Corollary 15. Let $(X, M, *)$ be an FM-space. Let $A_{1}, A_{2}, \ldots, A_{n}$, $S_{1}, S_{2}, \ldots, S_{n}, P$ and $Q$ be mappings from $X$ into itself satisfy (14.2) with $a=0$.

If conditions (14.1) 1nd (14.3) are satisfied then conclusions (i) and (ii) of Theorem 14 hold. Further if conditions (14.4) and (14.5) are satisfied then conclusion (iii) of Theorem 14 holds.

Remark 7. Since weak compatibility of two mappings is equivalent to their $R$-weak commutativity at their coincidence points. So we can replace condition of weak compatibility of mappings by $R$-weak commutativity at coincidence points in above theorems and corollaries.

\section{Discussions and Auxilary Results}

In view of above results it is very much clear that we extend, improve and generalize many results in metric spaces and fuzzy metric spaces. We prove common fixed point theorems for finite number of mappings 
in fuzzy metric spaces. This is the first effort in existing literature. To prove common fixed point theorems for contractive type condition with more than four mappings some commutative conditions for mappings are always essential. How many commutative conditions are necessary? As an answer of this question we are giving the following formulas:

(i) If the number of mappings are even and finite in above theorems and corollaries then there will be $\frac{n^{2}-2 n-8}{4}$ commutativity conditions, where $n=4,6,8,10,12, \ldots$ up to finite values. For example if $n=10$ then 18 commutativity conditions are required. (See (1.4)).

(ii) If the number of mappings are odd and finite in above theorems and corollaries then there will be $\frac{n^{2}-9}{4}$ commutativity conditions, where $n=5,7,9,11, \ldots$ up to finite values. For example if $n=7$ then 10 commutativity conditions are required. (See (7.4)).

(iii) If $n=1,2,3,4$ then any commutativity condition is not required. See Remark 4(v), 4(vi) and Corollaries 9-13.

Our results apply to a wider class of mappings than the results on compatible or compatible of type $(\alpha)$ or compatible of type $(\beta)$ maps since compatible or compatible of type $(\alpha)$ or compatible of type $(\beta)$ maps constitute a proper subclass of weakly compatible maps.

We point out that common fixed point theorems for finite number of maps can be proved without continuity of any mappings.

In our all results we replace the completeness of the whole space with a set of alternative conditions.

In this way we prove common fixed point theorems for finite number of maps in fuzzy metric spaces by relaxing, replacing and omitting some conditions in the analogous results.

Our results contain so many results in the existing literature and will be helpful for the workers in the field.

\section{REFERENCES}

[1] Y.J. Cho, Fixed point in fuzzy metric spaces, J. Fuzzy Math., 5(4) (1997), 949-962.

[2] Y.J. Cho, H.K. Pathak, S.M. Kang and J.S. Jung, Common fixed points of compatible maps of type $(\beta)$ on fuzzy metric spaces, Fuzzy Sets and Systems, 93 (1998), 99-111.

[3] A. George and P. Veeramani, On some results in fuzzy metric spaces, Fuzzy Sets and Systems, 64 (1994), 395-399.

[4] M.Grabiec, Fixed points in fuzzy metric spaces, Fuzzy Sets and Systems, 27 (1988), 385-389. 
[5] K. Iseki, Some applications of Banach type contraction principles, Math. Sem. Notes, Kobe Univ., 4 (1976), 211-214.

[6] I. Istratescu, A fixed point theorem for mappings with a probabilistic contractive iterated, Rev. Roumarie Math. Pure Appl., 26 (1981), 431-435.

[7] G. Jungck, Commuting mappings and fixed points, Ammer Math. Monthly, 83(1976), 261-263.

[8] G. Jungck, Compatible mappings and common fixed points, Internat. J. Math. Math. Sci., 9 (1986), 771-779.

[9] G. Jungck, Common fixed points for commuting and compatible maps on compacta, Pro. Amer. Math. Soc., 103 (1988), 977-983.

[10] G. Jungck, P.P. Murthy and Y.J. Cho, Compatible mappings of type $(A)$ and common fixed points, Math. Japonica, 38 (1993), 381-390.

[11] G. Jungck and B.E. Rhoades, Fixed point for set valued functions without continuity, Indian J. Pure Appl. Math., 29(3) (1998), 227-238.

[12] I. Kramosil and J. Michlek, Fuzzy metric and statistical metric spaces, Kybernetica, 11 (1975), 336-344.

[13] S.N. Mishra, N. Sharma and S.L. Singh, Common fixed points of maps on fuzzy metric spaces, Internat. J. Math. Sci., 17 (1994), 253-258.

[14] R.P. Pant, Common fixed points of noncommuting mappings, J. Math. Anal. Appl., 188 (1994), 436-440.

[15] R.P. Pant, Common fixed point theorems for contractive maps, J. Math. Anal. Appl., 226 (1998), 251-258.

[16] R.P. Pant, Common fixed points of Lipschitz type mappings pair, J. Math. Anal. Appl., 240 (1999), 280-283.

[17] R.P. Pant, Discontinuity and fixed points, J. Math. Anal. Appl., 240 (1999), 284-289.

[18] H.K. Pathak, Y.J. Cho, S.S. Chang and S.M. Kang, Compatible mappings of type $(P)$ and fixed point theorems in metric spaces and probabilistic metric spaces, Novi Sad J. Math., 26(2) (1996), 87-109.

[19] B.E. Rhoades, A comparison of various definitions of contractive mappings, Trans. Amer. Math. Soc., 226 (1977), 257-290.

[20] B.E. Rhoades, Contractive definition revisited, Contemp. Math., 21 (1983), 189-205.

[21] B. Schweizer and A. Sklar, Statistical metric spaces, Pac. J. Math., 10 (1960), $313-334$.

[22] S. Sessa, On a weak commutativity condition of mappings in fixed point consideration, Publ. Inst. Math. (Beogavd), 32(46) (1982), 149-155.

[23] S. Sessa, B.E. Rhoades and M.S. Khan, On common fixed points of compatible mappings in metric and Banach spaces, Internat. J. Math. Sci., 11(2) (1988), 375-392.

[24] S. Sharma, Common fixed point theorems in fuzzy metric spaces, Fuzzy Sets and Systems, 125 (2001), 1-8.

[25] S. Sharma, On fuzzy metric spaces, SEA Bull. of Math., 6(1) (2001), 147-157. 
[26] S. Sharma and B. Deshpande, Common fixed points of compatible maps of type ( $\beta$ ) on fuzzy metric spaces, Demonstratio Mathematica, XXXV(1) (2002), $165-175$.

[27] S. Sharma and B. Deshpande, Common fixed point for weakly compatible mappings without continuity in fuzzy metric spaces, EAMJ, 18(2) (2002), 183-193.

[28] S. Sharma and B. Deshpande, Discontinuity and weak compatibility in fixed point consideration on noncomplete fuzzy metric spaces, J. Fuzzy Math., 11(3) (2003), 671-683.

[29] S. Sharma and B. Deshpande, Common fixed point without continuity in fuzzy metric spaces, J. Korea Soc. Math. Edu. Ser B. Pure Appl. Math., 12(4) (2005), 289-306.

[30] S.P. Singh, Some common fixed point theorems in L spaces, Math. Sem. Notes, Kobe Univ., 7 (1979), 91-97.

[31] S.L. Singh and S. Kasahara, On some recent results on common fixed points, Indian J. Pure Appl. Math., 13 (1982), 757-761; corrigendum 14 (1983), 1075.

[32] S.L. Singh and B. Ram, Common fixed points of commuting mappings in 2metric spaces, Math. Sem. Notes Kobe Univ., 10 (1982),197-208.

[33] B.M.L. Tiwari and S.L. Singh, A note on recent generalization of Jungck contraction principle, J. UPGG. Acad. Soc., 3 (1986), 13-18.

[34] R. Vasuki, Common fixed point for R-weakly commuting maps in fuzzy metric spaces, Indian J. Pure Appl. Math., 30(4) (1999), 419-423.

[35] L.A. Zadeh, Fuzzy Sets, Inform Control, 8 (1965), 338-353.

\author{
Sushil SHARMA \\ Department of Mathematics \\ MADHAV VIGYAN MAHAVIDYALAY \\ UJJAIN (M.P.) \\ INDIA \\ E-mail address: sksharma20050yahoo.com
}

Bhavana Deshrande

DEPARTMENT OF MATHEMATiCS

Govt. Arts and Science P.G. College

RATLAM-457001 (M.P.)

INDIA

E-mail address: bhavnadeshpande@yahoo.com

Current address:

Bhavana Deshpande

"Sukhakarta"

90-Rajiv Nagar (Near Kasturba Nagar)

Ratlam-457001 (M. P.)

India

E-mail address: bhavnadeshpande@yahoo.com 